PHYSICAL REVIEW A 73, 029907(E) (2006)

\title{
Erratum: Generation of entangled states in cavity QED [Phys. Rev. A 72, 034304 (2005)]
}

Liu Ye, Long-Bao Yu, and Guang-Can Guo

(Received 26 January 2006; published 23 February 2006)

DOI: 10.1103/PhysRevA.73.029907

PACS number(s): 03.67.Mn, 42.50.Pq, 42.50.Vk, 99.10.Cd

In the above publication, the third term of Eq. (19) has a sign error because of our carelessness. This mistake makes the scheme impossible to generate a four-atom entangled cluster state.

We would also like to use this opportunity to thank Dr. Xin-Wen Wang for pointing this out. 